\title{
Fuzzy logic research work in Mexico motivated by Lotfi Zadeh
}

\author{
Oscar Castillo $^{1}$ and Patricia Melin ${ }^{1}$ \\ ${ }^{1}$ Tijuana Institute of Technology \\ Tijuana, Mexico
}

Received: 3 March 2021

Accepted: 8 June 2021

\begin{abstract}
We provide in this article a short review of the research work that has been done in Mexico on developing new methods and theory for designing intelligent systems utilizing type2 fuzzy systems in combination with soft computing techniques. Soft Computing (SC) is an area formed by intelligent paradigms, like fuzzy systems, neural networks, and bio-inspired and swarm algorithms, which may be utilized to build high performance hybrid systems. The combination of type-2 fuzzy systems with SC enables the constructing of efficient intelligent systems for solving complex problems in a wide diversity of areas, such as control, pattern recognition, medical diagnosis and others. We also recall some of the main moments and memories of encounters and meetings with the father of fuzzy logic (Prof. L. Zadeh), which were very positive and motivated us to continue his work and legacy.
\end{abstract}

Keywords: Fuzzy logic, Type-2 fuzzy logic, Fuzzy systems.

2020 Mathematics Subject Classification: $03 E 72$.

\section{Introduction}

Fuzzy logic is an area of soft computing that enables a system to reason with uncertainty [1]. The structure of a fuzzy system is comprised by a set of if-then rules defined as a function of fuzzy sets, an inference mechanism, and a set of parameterized membership functions. Fuzzy sets elevate the construct of a traditional set by enabling the membership degree to be any real value between 0 and 1 . Fuzzy systems have been utilized with relative success to numerous problems in a wide range of areas, such as decision-making, control, medical diagnosis, pattern recognition and classification. However, in real complex problems, the knowledge that is 
utilized to construct these fuzzy rules is uncertain, and type-1 fuzzy systems, whose membership functions are type-1, are not capable of handling such uncertainties. We also mention in this paper, type-2 fuzzy systems, in which the antecedent or consequent membership functions are type-2 fuzzy sets [2]. These fuzzy sets have membership degrees that themselves are type-1. They are very useful in situations when it is difficult to find a precise membership function for a fuzzy set. We have to say that to the moment, there have been around 78000 papers published on fuzzy logic worldwide, and around 1400 are from Mexico, but many are coming due to the encouraging words of Lotfi Zadeh.

Uncertainty is an intrinsic part of the intelligent systems that are utilized in real problems. The utilization of novel methods for management of incomplete information is of essential relevance. Type-1 fuzzy sets used in conventional fuzzy systems cannot fully handle the uncertainties present in intelligent systems [3]. Type-2 fuzzy sets that are utilized in type-2 fuzzy systems are able to handle such uncertainties in a more appropriate way. This paper overviews the use of fuzzy systems defined in terms of interval type-2 fuzzy logic for reducing the effects of uncertainty generated by the instruments, dynamic changing environments, external perturbations and noise, etc., for applications in control and pattern recognition.

\section{Summary of research work on type-2 fuzzy logic in control}

We have performed work on the design of type-2 fuzzy logic controllers using genetic algorithms and bio-inspired optimization methods [3]. In this section we offer an overview of some of the works that have been published utilizing type-2 fuzzy logic for control applications.

As a first example, we have considered the design of type-2 fuzzy systems for the longitudinal control of an F-14 airplane using genetic algorithms [4]. The longitudinal control is achieved by using only the elevators of the airplane. To achieve such control, it is required to use three input variables: stick, the elevation rate and the attack angle. These variables are the inputs to the fuzzy system, and then we can achieve controlling the output value of the elevators for stabilizing the airplane.

Another case is the use of an evolutionary algorithm for the optimal design of type-2 fuzzy reactive and tracking controllers for the autonomous navigation of mobile robots. The algorithm optimizes the type- 2 fuzzy systems for the two controllers. The reactive and tracking controllers are required to obtain the global navigation of the mobile robot in uncertain environments.

The use of novel methods for handling incomplete information is of essential relevance in applications. We have also considered the simulation of the effects of uncertainty produced by the instrumentation elements in type-1 and type-2 fuzzy logic controllers to perform a comparative analysis of the systems' response, in the presence of uncertainty [4]. This article presented a novel approach to optimize interval type-2 membership functions utilizing the human evolutionary model.

We have also considered the use of Ant Colony Optimization (ACO) for the ball and beam control problem, in particular for the problem of tuning a fuzzy controller of Sugeno type [4]. We have also considered the application of a simple ACO as a method of optimization for 
membership functions' parameters of a type-2 fuzzy logic controller in order to find the optimal intelligent controller for an autonomous wheeled mobile robot [4].

We have also used the Particle Swarm Optimization (PSO) method to find the parameters of the membership functions of a type-2 fuzzy logic controller (Type-2 FLC) in order to minimize the state error for linear systems [4]. PSO is utilized for finding the optimal Type-2 FLC to obtain the regulation of the output and the system stability.

In general, the above-mentioned applications cases are representative of the state of art in control. However, we also have to mention that there exist applications of type- 2 fuzzy logic in pattern recognition, time series prediction, and classification, which have been successful in the real world, but are not the main concern in this paper $[5,6]$. There have also been important theoretical advances on type-2 fuzzy logic based on the original ideas of Zadeh [7-9] that have enabled more efficient processing and type reduction, which have helped obtaining solutions to real world problems. Finally, we have to say that there are many more recent works that have been done in applying fuzzy logic to improve the performance of bioinspired optimization techniques [10-14].

\section{Type-2 fuzzy logic applications in pattern recognition}

One approach that we have worked on in our research group for face recognition uses modular neural networks with a fuzzy logic method for response integration [5]. The response integration method is based on a proposed type-2 fuzzy Sugeno integral. Response integration is necessary to properly combine the outputs of the modules in the modular network.

Another approach has been the use of neural networks, fuzzy logic and genetic algorithms for voice recognition [5]. In particular, we have considered the case of speaker recognition by performing an analysis of the sound signals with the help of neural and fuzzy systems, to verify if the human speaker can be recognized. The obtained recognition rates with the proposed approach are very good.

We have also considered the use of three modular neural networks as systems for recognizing persons based on the iris biometric measurement of humans [6]. In these systems, image processing methods are used to improve the human iris database. Simulation results with real iris databases were used the test the proposed modular network variant.

We also have worked on human recognition from ear images as biometric using modular neural networks with preprocessing ear images as network inputs [6]. In this case, a modular neural network structure formed by twelve modules was proposed. This was done in order to simplify the problem, and in this way to obtain accurate recognition results.

We have also proposed a new approach for human recognition using as information the combination of three biometric measures: iris, ear, and voice of a person [6]. The final decision is formed with the results of the three modules and uses a type-2 fuzzy system for uncertainty handling in the decision-making process. There are also more recent works on applying type-2 fuzzy logic in pattern recognition and medical diagnosis [15-20]. 


\section{Motivation by Prof. Zadeh's work}

The motivating and inspiring ideas, as well as the research work of Prof. Zadeh have been fundamental in the research work that has been done in Mexico [7, 8, 9]. He did always support our research group's work and he was very kind to accept our invitation to deliver a keynote lecture at the World IFSA 2007 Congress that was organized in Cancun, Mexico in 2007. This was an important lecture, especially for Latin America and Mexico professors, researchers and students. In Fig. 1, we can find the arrival of Prof. Zadeh to the opening ceremony of the IFSA 2007 congress.

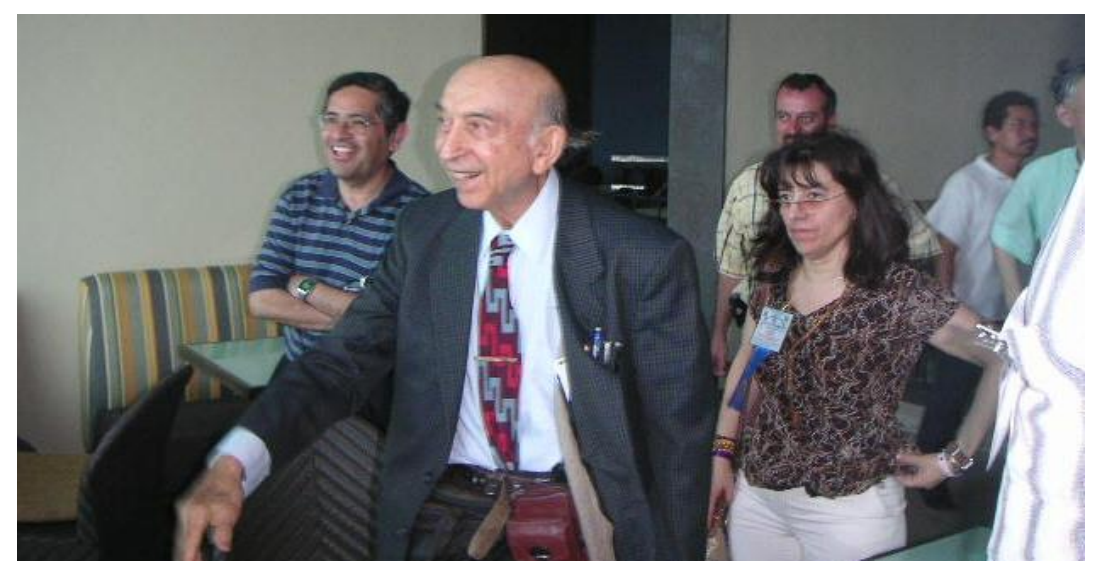

Figure 1. Arrival of Prof. Zadeh to the IFSA 2007 World Congress

In Fig. 2, we can find a photo of Prof. Zadeh with the classical Mexican hat during the banquet of IFSA 2007. In Fig. 3, we can find a photo of Prof. Zadeh receiving an Award from Prof. Melin during the banquet of IFSA 2007.

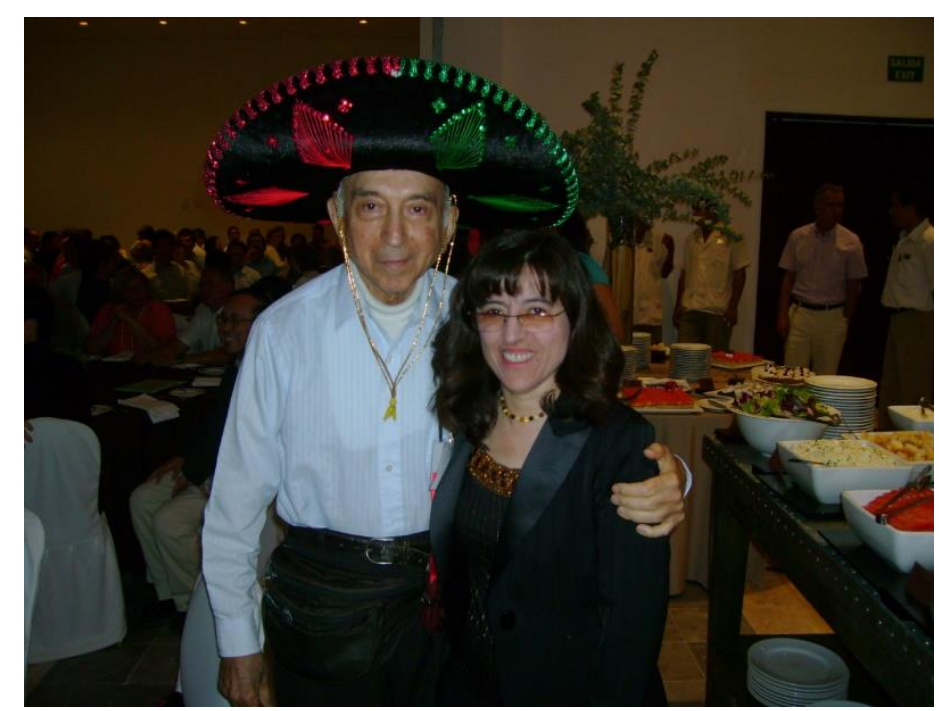

Figure 2. Prof. Zadeh with the traditional Mexican hat, which was a gift from the organizers of the IFSA 2007 Congress 


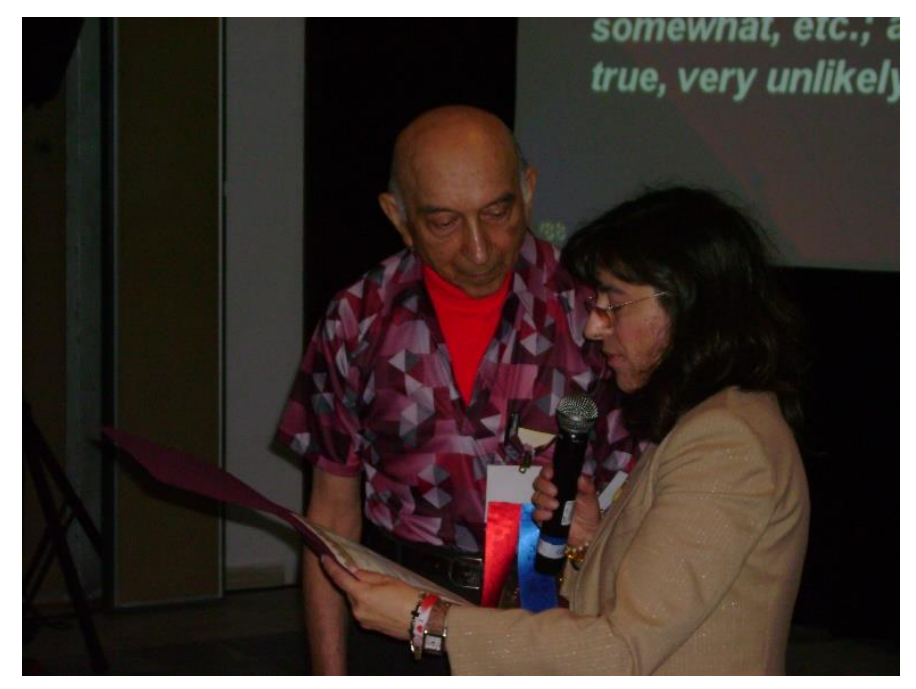

Figure 3. Prof. Zadeh receiving an award during the IFSA 2007 banquet

On the other hand, we have to say that our research group at the Tijuana Institute of Technology, being right at the border with the United States, has the advantage of being very close to Berkeley, where Prof. Zadeh used to live. Actually, for several years there were annual conferences organized in Berkeley or San Francisco, and we had the pleasure to attend and participate together with many of our students. We always admire that Prof. Zadeh was very happy to see our students and talk to them and even took pictures with them. In Fig. 4, a photo at the awards ceremony of the BISC 2005 conference is shown, where Patricia Melin is with Professors Zadeh, Zadrosny and Pedrycz.

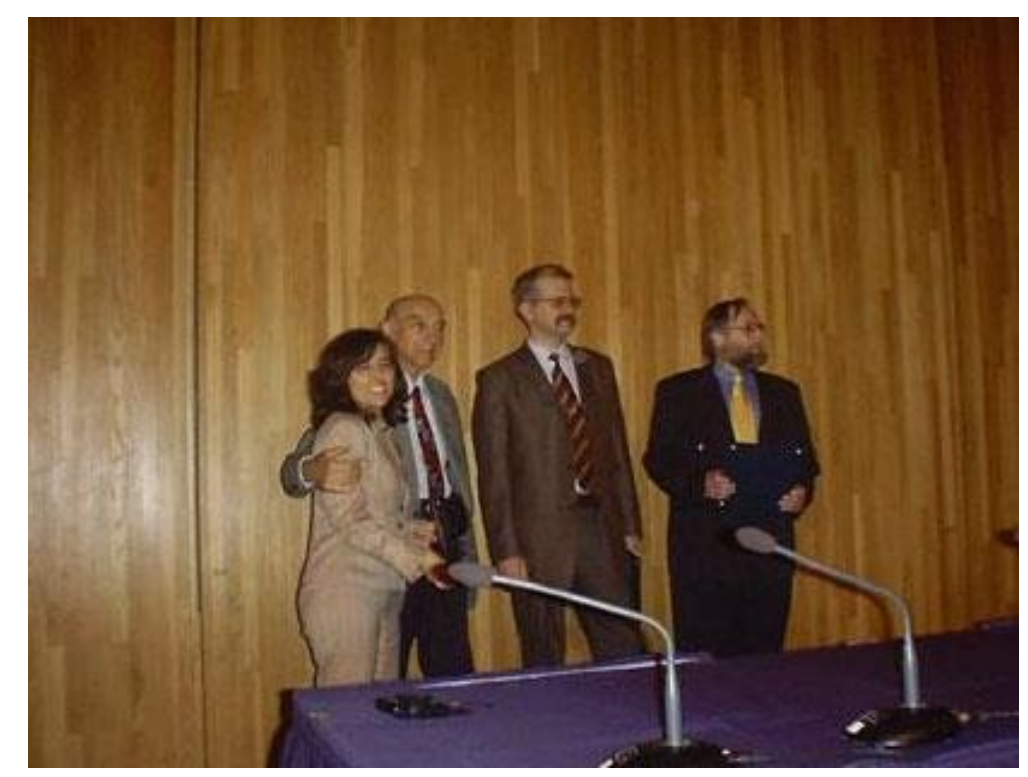

Figure 4. Photo from the BISC 2005 conference in Berkeley 
In Fig. 5, we can find a more recent encounter during the World conference on Soft Computing in Berkeley in 2014. Also, in May 2016 we attended a conference in Berkeley. The photos in Fig. 6 and Fig. 7 were taken there.

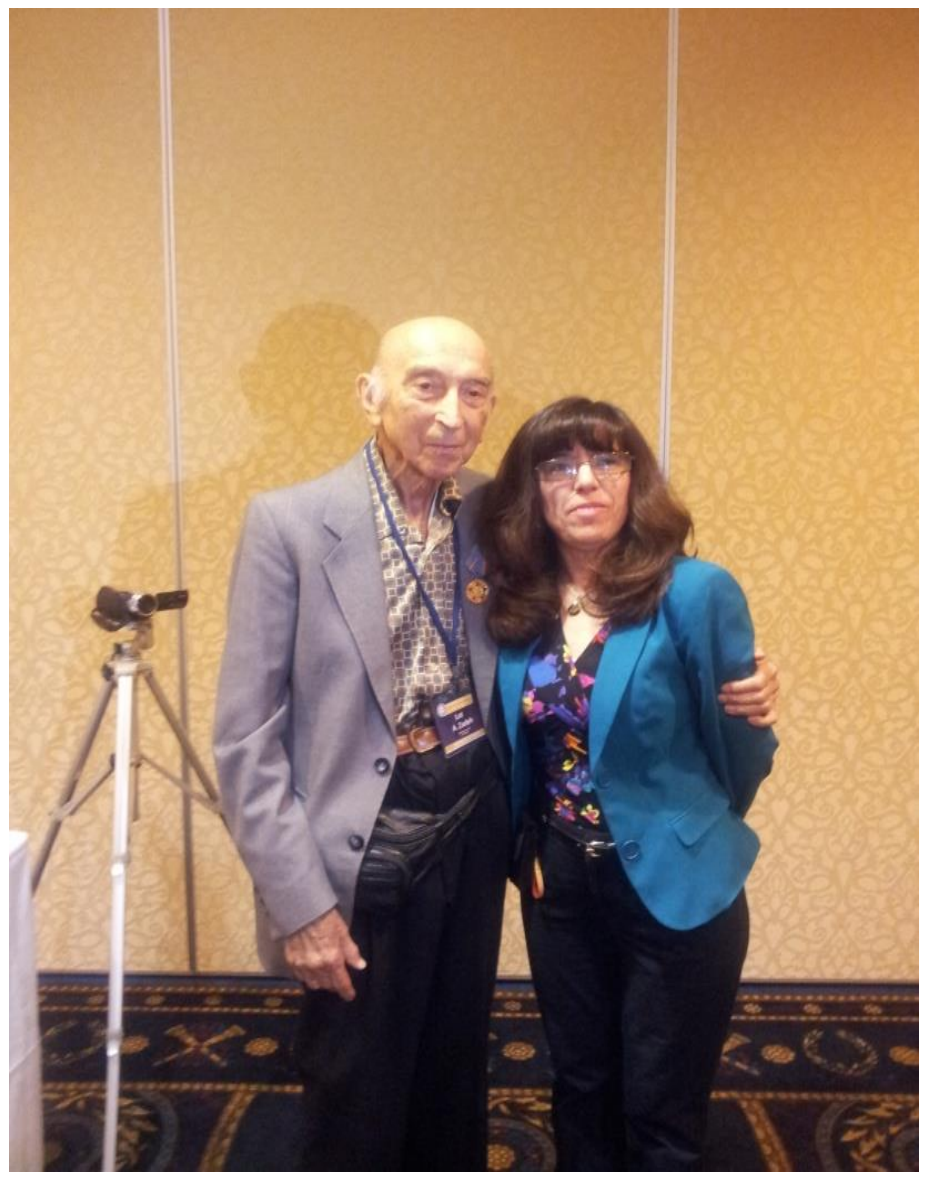

Figure 5. Soft Computing conference in Berkeley in 2014

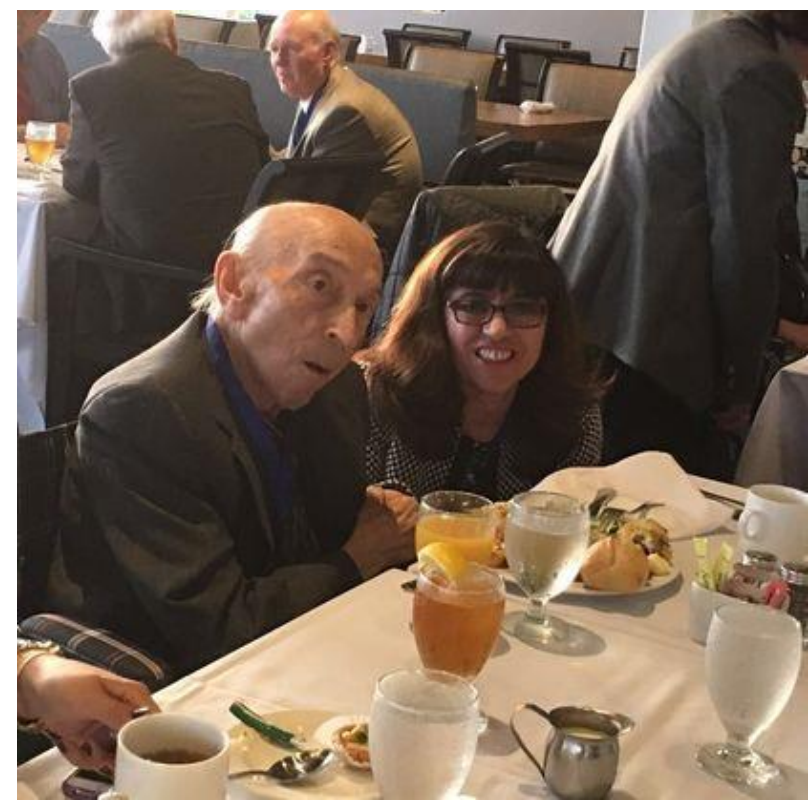

Figure 6. Photo at the banquet of the conference in Berkeley 


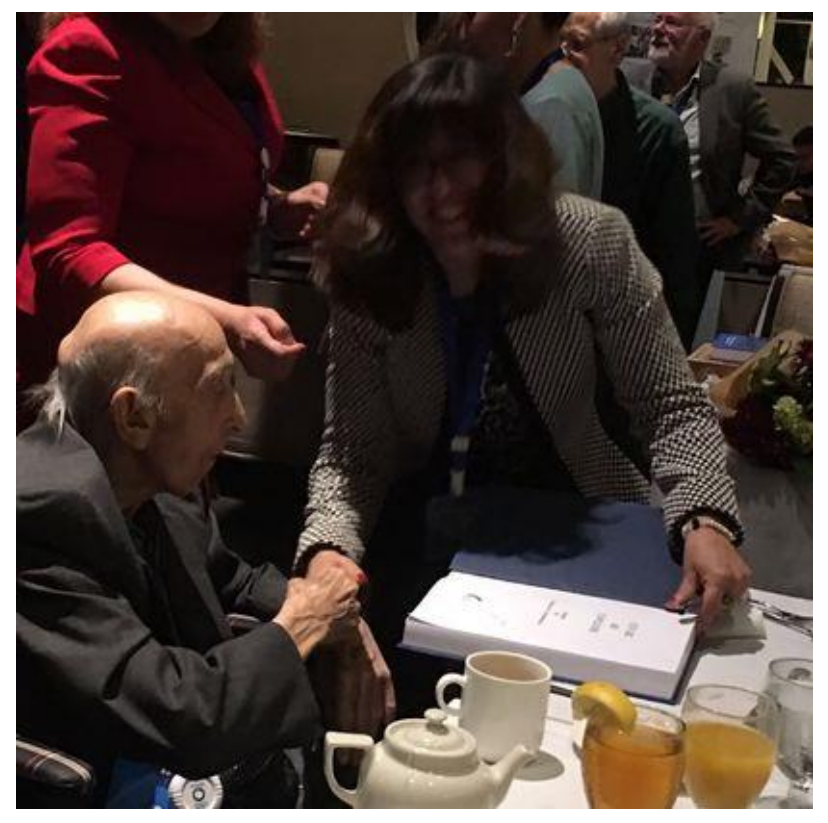

Figure 7. Prof. Melin asking for Prof. Zadeh's signing of the commemorative book

In Fig. 8, a photo of Prof. Zadeh with Oscar Castillo in Berkeley is shown. A photo with graduate students in 2016 is shown in Fig. 9.

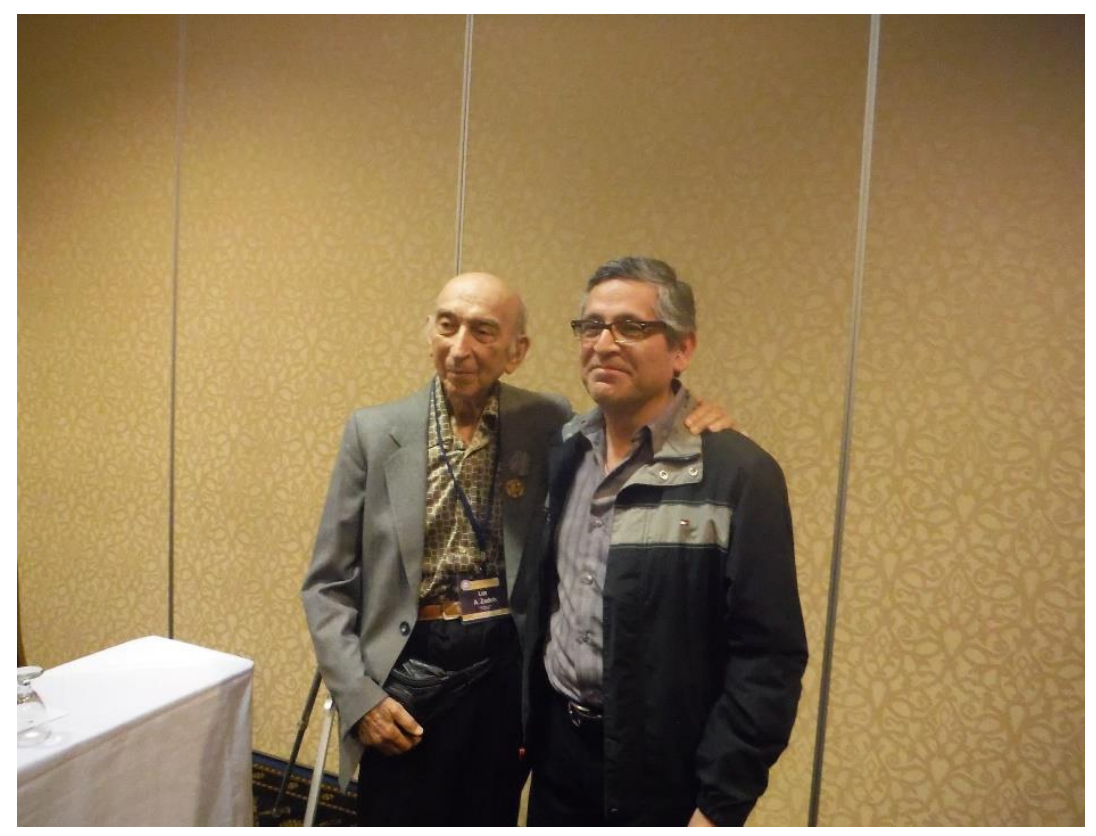

Figure 8. Prof. Zadeh with Oscar Castillo in Berkeley in 2014 


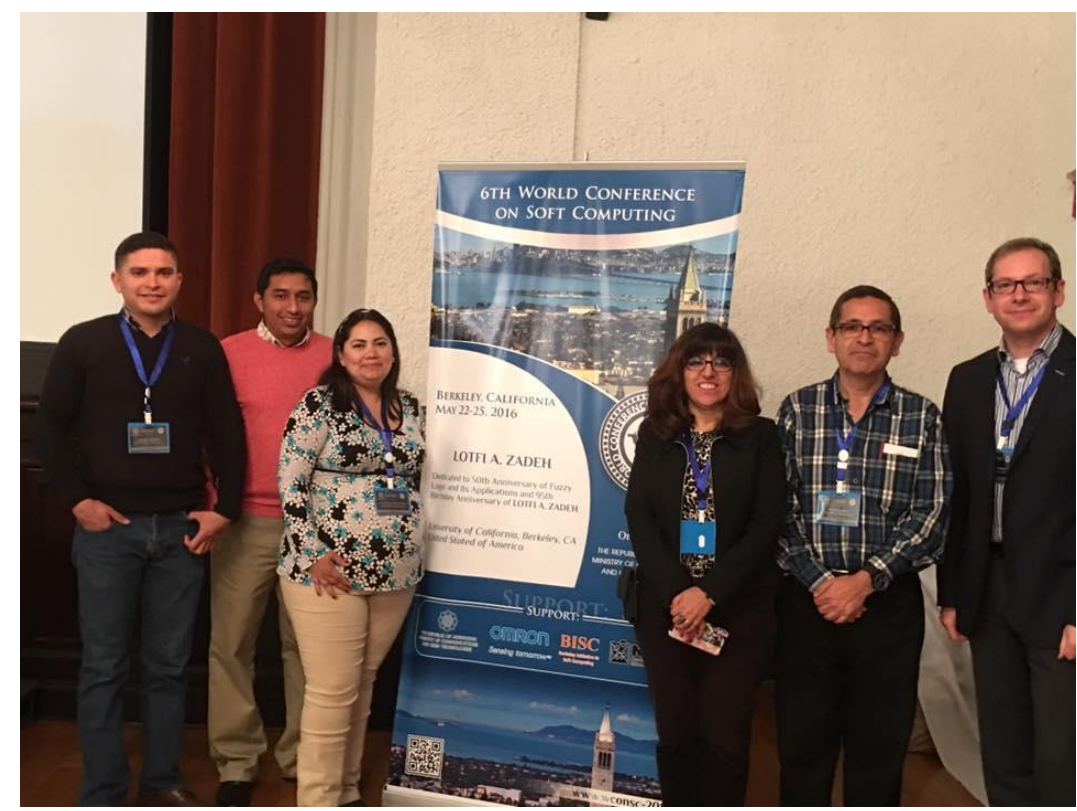

Figure 9. A photo of graduate students of Patricia Melin and Oscar Castillo with Prof. Reformat in Berkeley in 2016

In Fig. 10, we can find one of the last keynote lectures of Prof. Zadeh, along with Prof. Janusz Kacprzyk in Berkeley (May 22, 2016).

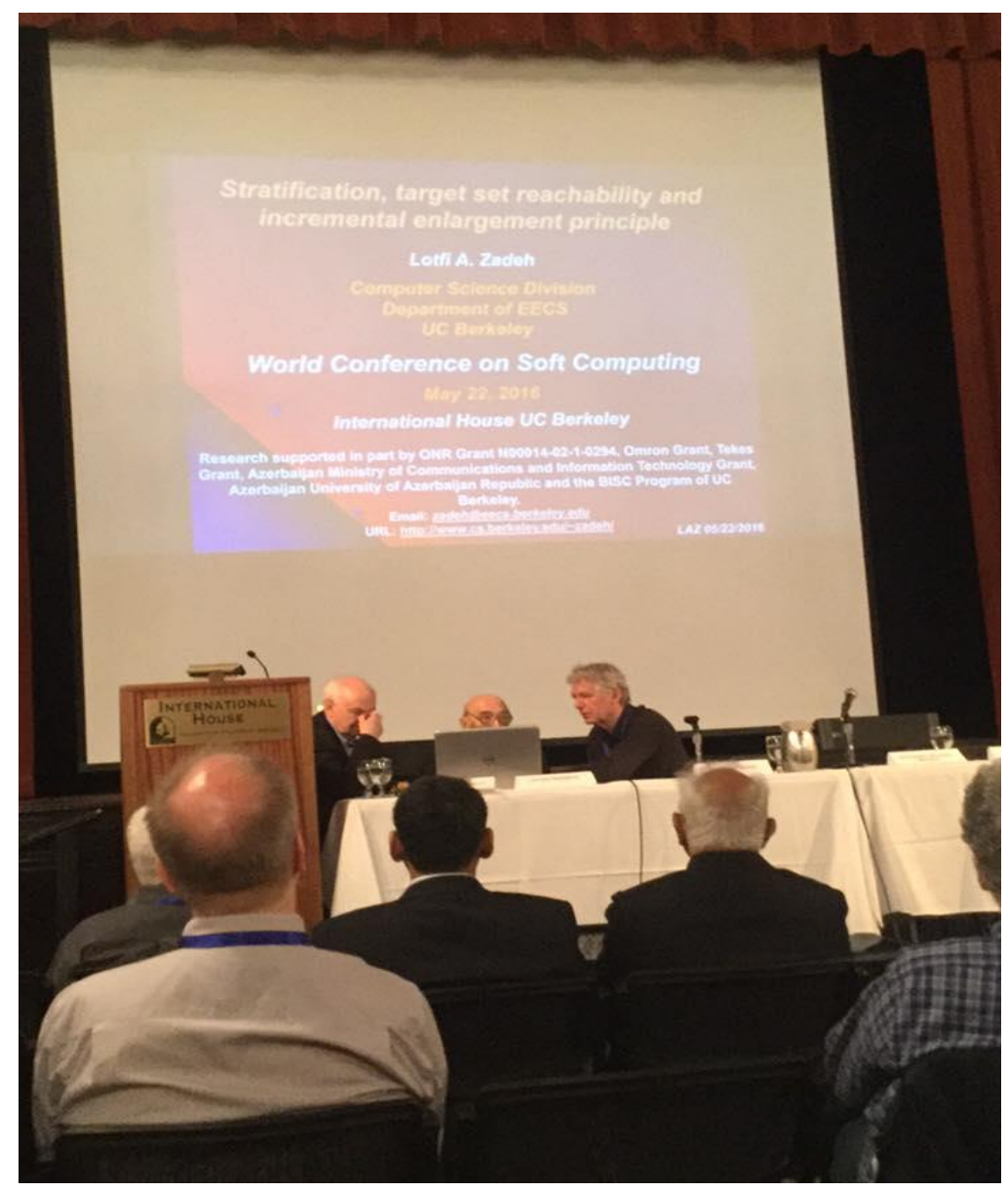

Figure 10. Keynote lecture of Prof. Zadeh in 2016 
Type-2 fuzzy logic was always endorsed and motivated by Prof. Zadeh as he was the first one to propose the novel idea of type-2 fuzzy sets. In this sense, he has always supported and encouraged the works of the main researchers (and their students) in this area, like Professors J. M. Mendel, R. John, H. Hagras, and others, like the authors of this paper. It is our strong opinion, that type-2 fuzzy logic is one of the most important and relevant areas in fuzzy logic, and we believe that many researchers in the near future will be working on many open problems, both theoretical and in the applications.

\section{Conclusions}

In this article, a brief overview of the novel methods for constructing intelligent systems using type-2 fuzzy logic and SC has been presented. In this article, the utilization of fuzzy logic of higher order has been presented, which has been named type-2 fuzzy logic, but in the future could be elevated to type-3 and to type-n. In addition, the application of metaheuristics optimization techniques, like GAs, PSO and ACO, has been proposed to automatically and optimally design type-2 fuzzy systems for intelligent control and pattern recognition applications. Finally, we also offered an account of some of the encounters with Prof. Zadeh in several Berkeley meetings and conferences, where we had discussions and meetings with him and his good colleagues and friends, like Prof. Janusz Kacprzyk, Prof. Ronald Yager, Prof. Marek Reformat, Prof. Witold Pedrycz, Prof. Jim Keller, Prof. Jerry Mendel and others.

\section{References}

[1] Castillo, O., \& Melin, P. (2001). Soft Computing for Control of Non-Linear Dynamical Systems. Springer-Verlag, Heidelberg, Germany.

[2] Castillo, O., \& Melin, P. (2003). Soft Computing and Fractal Theory for Intelligent Manufacturing. Springer-Verlag, Heidelberg, Germany.

[3] Castillo, O., \& Melin, P. (2008). Type-2 Fuzzy Logic: Theory and Applications. SpringerVerlag, Heidelberg, Germany.

[4] Castillo, O. (2012). Type-2 Fuzzy Logic in Intelligent Control Applications. SpringerVerlag, Heidelberg, Germany.

[5] Melin, P., \& Castillo, O. (2002). Modelling, Simulation and Control of Non-Linear Dynamical Systems. Taylor and Francis, London, Great Britain.

[6] Melin, P., \& Castillo, O. (2005). Hybrid Intelligent Systems for Pattern Recognition. Springer-Verlag, Heidelberg, Germany.

[7] Zadeh, L. A. (1996). Fuzzy Logic = Computing with Words. IEEE Transactions on Fuzzy Systems, Vol. 4, No. 2, May 1996, 103.

[8] Zadeh, L. A. (1989). Knowledge representation in Fuzzy Logic. IEEE Transactions on knowledge data engineering, Vol. 1, p. 89. 
[9] Zadeh, L.A. (1998). Fuzzy Logic. Computer, Vol. 1, No. 4, 83-93.

[10] Castillo, O., \& Amador-Angulo, L. (2018). A generalized type-2 fuzzy logic approach for dynamic parameter adaptation in bee colony optimization applied to fuzzy controller design. Information Sciences 460-461, 476-496.

[11] Melin, P, Olivas, F, Castillo, O, Valdez, F, Soria, J, \& Garcia, J. (2013). Optimal design of fuzzy classification systems using PSO with dynamic parameter adaptation through fuzzy logic. Elsevier Exp Syst Appl, 40 (8): 3196-3206.

[12] Neyoy, H., Castillo, O., \& Soria, J. (2012). Dynamic fuzzy logic parameter tuning for ACO and its application in TSP Problems. Studies in Computational Intelligence 451, Springer, 259-271.

[13] Olivas, F., Valdez, F., Castillo, O. \& Melin, P. (2016). Dynamic parameter adaptation in particle swarm optimization using interval type-2 fuzzy logic. Soft Computing, 20(3), 1057-1070.

[14] Olivas, F., Valdez, F., Castillo, O. \& Melin P. (2019). Interval type-2 fuzzy logic for dynamic parameter adaptation in a modified gravitational search algorithm. Information Sciences 476, 159-175.

[15] Sanchez, D., Melin, P., Castillo, O. (2017). Optimization of modular granular neural networks using a firefly algorithm for human recognition. Eng. Appl. of AI 64: 172-186.

[16] González, B., Valdez, F., Melin, P., \& Prado-Arechiga, G. (2015). Fuzzy logic in the gravitational search algorithm for the optimization of modular neural networks in pattern recognition. Expert Systems with Applications, 42 (14), 5839-5847.

[17] González, B., Valdez, F., Melin, P., \& Prado-Arechiga, G. (2015). Fuzzy logic in the gravitational search algorithm enhanced using fuzzy logic with dynamic alpha parameter value adaptation for the optimization of modular neural networks in echocardiogram recognition. Applied Soft Computing 37, 245--254.

[18] Miramontes, I., Guzman, J., Melin, P., \& Prado-Arechiga, G. (2018). Optimal Design of Interval Type-2 Fuzzy Rate Level Classification Systems Using the Bird Swarm Algorithm. Algorithms, 11 (12), 206.

[19] Ontiveros, E., Melin, P., \& Castillo, O. (2018). High order $\alpha$-planes integration: A new approach to computational cost reduction of General Type-2 Fuzzy Systems. Eng. Appl. Artif. Intell., 74: 186-197.

[20] Sánchez, D., \& Melin, P. (2014). Optimization of modular granular neural networks using hierarchical genetic algorithms for human recognition using the ear biometric measure. Engineering Applications of Artificial Intelligence, 27, 41-56. 